\title{
Incorporação de ômega-3 no tecido muscular da tilápia do Nilo alimentada com dietas contendo silagem de cabeça de camarão
}

\author{
Incorporation of n-3 in muscular tissue of Nile tilapia fed with shrimp head silage
}

\author{
Carolina Nunes Costa ${ }^{\mathrm{III}}$ Jaff Ribeiro da Silva ${ }^{\mathrm{II}}$ Fulvio Viegas Teixeira de Melo ${ }^{\mathrm{II}}$ Hamilton Hisano ${ }^{\mathrm{II}}$ \\ Janice Izabel Druzian" Leandro Portz ${ }^{\text {IV }}$
}

\section{RESUMO}

\begin{abstract}
$O$ objetivo deste estudo foi avaliar a incorporação de ômega-3 EPA (eicosapentaenóico) e DHA (docosahexanóico) no tecido muscular de tilápias do Nilo alimentadas com dietas suplementadas com diferentes níveis de silagem de cabeça de camarão Litopenaeus vannamei $(0$, $4,8,12,16 \%)$. Foram analisados os teores de EPA, DHA $e$ lipídios totais das dietas e dos tecidos musculares das tilápias. Os teores de lipídios totais variaram entre 6,70 a 10,30\% nas dietas e 0,79 a 1,37\% no tecido muscular. Através da análise de regressão, houve um aumento nos teores de ômega-3 $(r=0,92)$ com a inclusão da silagem na dieta. Foi observada incorporação de 0,59\% (1,34mg $\left.100 \mathrm{~g}^{-1}\right)$ e $9,5 \%$ (40,50mg $\left.100 \mathrm{~g}^{-1}\right)$ de EPA e DHA, respectivamente, no tratamento com $16 \%$ de silagem na dieta. A inclusão de $16 \%$ da silagem ácida de cabeça de camarão na dieta das tilápias aumentou significativamente os níveis de incorporação de EPA e DHA no filé.
\end{abstract}

Palavras-chave: silagem, nutrição, Litopenaeus vannamei, tilápia, Oreochromis niloticus.

\section{ABSTRACT}

The aim of the present study was to assess the effect of diets supplemented with shrimp-head silage on the incorporation of $n-3$ in the Nile tilapia fillets. EPA (eicosapentaenoic), DHA (docosahexanoic) and total lipids were determined by chromatography in feeds and the muscles of tilapia specimens were submitted to diets supplemented with a varying degree of silage $(0,4,8,12,16 \%)$. The total lipid content ranged from 6.70 to $10.30 \%$ in the diets and from 0.79 to $1.37 \%$ in the tilapia fillets. In diet with high concentration of silage, it was observed an increase in the level of omega-3 $(r=0.92)$. There was an incorporation of $0.59 \%\left(1.34 \mathrm{mg}_{\left.100 \mathrm{~g}^{-1}\right)}\right.$ and $9.5 \%\left(40.50 \mathrm{mg}^{\left.100 \mathrm{~g}^{-1}\right)}\right.$ of EPA and DHA, respectively, when the addition of silage to the diet was $16 \%$. The results implication states that the inclusion of $16 \%$ of the shrimp head silage in the diet for tilapias increases the levels of incorporation of EPA and DHA in the filet.

Key words: silage, nutrition, Litopenaeus vannamei, tilapia, Oreochromis niloticus.

\section{INTRODUÇÃO}

A carcinicultura brasileira produziu em 2009 cerca de 65 mil toneladas. Considerando que aproximadamente $33 \%$ do peso vivo do camarão sejam descartados no processamento industrial, estima-se que a produção de resíduos do beneficiamento nesse mesmo ano tenha sido de cerca de 21 mil t (MPA, 2010).

Uma forma simples e barata de aplicação racional de resíduos de pescado é a produção de silagem, pois, além de apresentar nível protéico próximo ao material residual original, também preserva os ácidos graxos de alto valor biológico (BORGHESI, 2004). O resíduo da indústria camaroneira é considerado uma fonte protéica de boa qualidade para alimentação de peixes (CAVALHEIRO et al., 2007).

As dietas fornecidas aos peixes exercem efeitos diretos sobre a composição muscular,

IDepartamento de Pesca e Aquicultura, Universidade Federal de Pernambuco (UFRPE), 52171-900, Recife, PE, Brasil. E-mail: carolncosta @ yahoo.com.br. Autor para correspondência.

"Faculdade de Farmácia, Universidade Federal da Bahia (UFBA), 40170-115, Salvador, BA, Brasil.

IIIEmbrapa Agropecuária Oeste, Dourados, MS, Brasil.

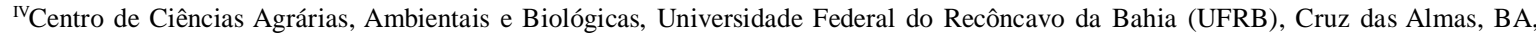
Brasil. 
especialmente no teor de lipídios e na composição de ácidos graxos (OM et al., 2001). Os lipídios, além de auxiliar o transporte de vitaminas lipossolúveis, são fontes de energia e de ácidos graxos essenciais. Sua importância em dietas para organismos aquáticos está associada ao aumento da palatabilidade, economia da proteína, melhora da textura do tecido muscular e perfil de ácidos graxos (MARTINO \& TAKAHASHI, 2001; MARTINO \& PORTZ, 2006).

Na composição de ácidos graxos, tem sido atribuída elevada importância nutricional aos ácidos da família ômega-3, especialmente, EPA e DHA. Nocaso do resíduo de camarão, além do elevado teor protéico, destaca-se também o alto teor de ácidos graxos ômega-3 que pode atingir $40 \%$ dos lipídios totais (KRZYNOWEK \& PANUNZIO, 1989). Peixes de água doce geralmente contêm baixas proporções de ômega-3, quando comparados aos peixes marinhos, em função da alimentação natural rica em ácidos graxos ômega-6, principalmente o ácido linoleico e o araquidônico (HOAR et al., 1979).

As indústrias alimentícias e farmacêuticas vêm desenvolvendo concentrados e produtos suplementados com ômega-3 (DAVID, 1993), sendo estes favoráveis à saúde humana, principalmente na prevenção de doenças cardiovasculares, dentre outras (STEFFENS, 1997).

O objetivo deste trabalho foi avaliar a incorporação de ácidos graxos ômega-3 EPA (eicosapentaenóico, 20:5n-3) e DHA (docosahexanóico, 22:6n-3) no tecido muscular da tilápia do Nilo, alimentada com dietas suplementadas contendo diferentes níveis de silagem de cabeça de camarão, considerando os teores de EPA, DHA e lipídios totais das dietas e filés.

\section{MATERIAL E MÉTODOS}

Resíduos da cabeça do camarão branco Litopenaeus vannamei in natura oriundos do processamento industrial foram moídos e, posteriormente, adicionou-se os ácidos fórmico e propiônico (1:1 v/v, 3\% v/p) e BHT como antioxidante $(0,02 \%)$. A biomassa foi mantida em recipientes de plástico, com capacidade para 20L, fechado para fermentação e periodicamente foi revolvida para sua homogeneização. $\mathrm{O}$ pH foi monitorado diariamente por meio de peagâmetro digital (HANNA H-112).

Foi utilizada, na elaboração das dietas, a silagem ácida com fermentação de 30 dias, com os seguintes resultados (amostra úmida): 14,8\% de proteína bruta; $2,38 \%$ de lipídios totais e 2,73\% de cinzas, segundo COSTA (2007). Cinco dietas isoprotéicas e isoenergéticas foram formuladas de acordo com exigências do NRC, variando o nível de inclusão da silagem ácida da cabeça do camarão (Tabela 1). As rações foram processadas em máquina de moer carne e, em seguida, secas em estufa de ventilação forçada por $24 \mathrm{~h} \mathrm{a} 60^{\circ} \mathrm{C}$.

Os alevinos de tilápia com peso médio de $7,2 \pm 0,5 \mathrm{~g}$ foram distribuídos aleatoriamente em 20 caixas de polietileno de 100L. Foram estocados 10 peixes em cada caixa, em sistema de recirculação de água. Os peixes foram alimentados com as diferentes dietas experimentais até a saciedade aparente, três vezes ao dia, durante 60 dias. Após esse período, todas as tilápias foram abatidas por superdosagem de benzocaína, posteriormente filetadas e estocadas a $-18^{\circ} \mathrm{C}$ até o momento das análises.

Para a extração dos lipídios totais das amostras das dietas e dos filés das tilápias foi utilizado o método de BLIGH \& DYER (1959). A fração lipídica foi submetida à esterificação segundo JOSEPH \& ACKMAN (1992). Os ésteres de ácidos graxos foram analisados em um cromatógrafo gasoso modelo VARIAN CP-3800, com detector de ionização de chama (GC-DIC). A coluna utilizada foi WAX $(25 \mathrm{mx} 0,25 \mathrm{~mm} \times 0,2 \mu \mathrm{m})$, com um fluxo de $1,3 \mathrm{ml} \mathrm{minutos}^{-1}$ de Hélio. As temperaturas do detector e injetor foram de $280^{\circ} \mathrm{C}\left(150^{\circ} \mathrm{C}\right.$ até $\left.230^{\circ} \mathrm{C}\right)$, com três rampas, totalizando 90 minutos de corrida. A identificação dos picos dos metil ésteres de ácidos graxos das amostras foi realizada por comparação de seus respectivos Tempos de retenção (Tr) com os de padrões de metil ésteres de EPA e DHA(Sigma/EUA). A quantificação foi realizada por normalização das áreas dos picos de EPA e DHA em relação à soma das áreas de todos os picos. Para os cálculos dos parâmetros das dietas e do tecido muscular das tilápias, foram utilizadas médias de extração em duplicatas dos lipídios totais e (EPA e DHA).

Os dados dos lipídios totais, EPA e DHA das dietas e dos filés das tilápias foram submetidos à análise de variância e, quando significativo $(\mathrm{P}<0,05)$, efetuou-se a análise de regressão, utilizando-se o aplicativo Sisvar v. 4.0. (FERREIRA, 2009).

\section{RESULTADOS E DISCUSSÃO}

Os teores de lipídios totais das dietas (Tabela 2) contendo diferentes níveis de silagem de cabeça de camarão nas diferentes formulações das dietas apresentaram efeito linear $\hat{Y}=4,16-0,095375 x$, $\mathrm{R}^{2}=0,99$. Quanto ao tecido muscular das tilápias, as médias dos lipídios totais variaram entre $0,79-1,37 \%$, e foi possível observar efeito linear $\hat{Y}=1,356-0,040250 x$, 
Tabela 1 - Ingredientes e composição das dietas formuladas com diferentes níveis de silagem ácida de resíduo do camarão.

\begin{tabular}{|c|c|c|c|c|c|}
\hline \multirow{2}{*}{ Ingredientes } & \multicolumn{5}{|c|}{ 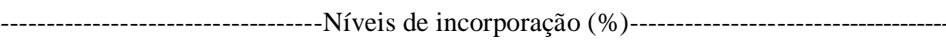 } \\
\hline & $\mathrm{T} 1$ & $\mathrm{~T} 2$ & $\mathrm{~T} 3$ & $\mathrm{~T} 4$ & T5 \\
\hline Silagem de camarão fermentada & 0 & 4,00 & 8,00 & 12,00 & 16,00 \\
\hline Farelo de soja & 66,00 & 60,37 & 54,58 & 48,80 & 43,15 \\
\hline Farelo de milho & 16,48 & 18,68 & 20,28 & 23,30 & 25,83 \\
\hline Farelo de trigo & 8,40 & 8,40 & 8,58 & 8,40 & 8,40 \\
\hline DL - Metionina (98\%) & 0,40 & 0,36 & 0,32 & 0,28 & 0,25 \\
\hline Cloreto de colina & 0,05 & 0,05 & 0,05 & 0,05 & 0,05 \\
\hline Fosfato bicálcico & 4,00 & 4,00 & 4,00 & 4,00 & 4,00 \\
\hline Mistura vitamínica $^{1}$ & 0,20 & 0,20 & 0,20 & 0,20 & 0,20 \\
\hline Mistura mineral $^{2}$ & 0,20 & 0,20 & 0,20 & 0,20 & 0,20 \\
\hline Óleo de soja & 4,30 & 3,70 & 3,32 & 2,60 & 1,90 \\
\hline BHT (antioxidante) & 0,02 & 0,02 & 0,02 & 0,02 & 0,02 \\
\hline Energia digestível $\left(\mathrm{kcal} \mathrm{g}^{-1}\right)$ & 3201,10 & $\begin{array}{l}\text { fo em Nut } \\
3199,07\end{array}$ & 3201,95 & 3201,40 & 3201,10 \\
\hline Proteína digestível (\%) & 30,02 & 30,05 & 30,00 & 30,00 & 30,05 \\
\hline
\end{tabular}

${ }^{1}$ Composição da mistura vitamínica/kg: Vitamina A - 6000UI, Colina - 54,2mg, Vitamina D3 - 2250UI, Niacina - 30mg, Biotina - 2mg, Vitamina B12 - 20mcg, Vitamina C - 192,5mg, Vitamina E - 75mg, vitamina K3 - 3mg, Ácido pantotênico - 30mg, Ácido fólico - 3mg, Piridoxina - 8mg, Riboflavina - 10mg, Tiamina - 5mg, Antioxidante - $1,98 \mathrm{mg}$.

${ }^{2}$ Composição da mistura mineral/kg: Selênio - 0,4mg, Cobre - 15mg, Zinco - 150mg, Manganês - 60mg, Iodo - 4,5mg, Cobalto - $2 \mathrm{mg}$, Ferro 100mg. *Cálculos realizados com base nos valores de energia e proteína digestível dos seguintes alimentos: farelo de soja, glúten de milho, milho e farelo de trigo (PEZZATO et al., 2002). Valores analisados de extrato etéreo (10,97\%) da silagem de camarão. Valores calculados de fibra bruta (CAVALHEIRO et al., 2007); energia digestível e proteína digestível (FAGBENRO \& BELLO-OLUSOJI, 1997). Os valores fornecidos em relação à composição dos nutrientes foram baseados em cálculos realizados.

$\mathbf{R}^{2}=0,94$. Os maiores acúmulos de lipídios totais no tecido muscular foram obtidos nas tilápias alimentadas com a dieta controle. Os resultados confirmaram que a composição lipídica dos peixes é reflexo direto da dieta oferecida, além de variar de acordo com a espécie (FURUYA et al., 2006).

Resultados semelhantes foram encontrados por VILA NOVA et al. (2005), que observaram valores de $0,99 \%$ de lipídio total no tecido muscular de tilápias do Nilo alimentadas com dietas comerciais. Em estudos realizados com dietas suplementadas com óleo de linhaça e óleo de girassol, VISENTAINER et al. (2005) observaram que o lipídio total no tecido muscular das tilápias variaram entre 1,0 a 1,2\%. Porém, em dietas ricas em óleos contendo sorgo e alta concentração de tanino, os níveis de lipídio total foram superiores ao encontrados no presente estudo, variando entre 3,50 a 4,20\% (AIURA\& CARVALHO 2004).

Não foram detectados EPA e DHA na dieta controle (sem incorporação de silagem), enquanto que a adição máxima de silagem (16\%) resultou na ração 0,59 e $9,45 \%$ de EPAe DHA, respectivamente. As dietas formuladas com teores maiores de silagem ácida de camarão demonstraram possuir quantidades expressivas do ácido graxo poliinsaturado DHA. Os percentuais dos ácidos graxos EPA $(0,74-3,07 \%$; 0,27-
$0,59 \%)$ e DHA $(0,76-3,78 \%-2,15-9,45 \%)$ obtidos nas frações de lipídios totais isolados das dietas e do tecido muscular das tilápias, respectivamente, foram quantificados por normalização das áreas dos picos (Tabela 2).

Segundo HUANG et al. (1998) e ROSENLUND et al. (2001), a composição dos ácidos graxos do tecido muscular dos peixes pode estar relacionado à composição de lipídios da dieta que estes consomem. De acordo com SRIKET et al. (2007), crustáceos possuem em sua composição corporal ácidos graxos altamente insaturados como EPA e DHA, corroborando os resultados encontrados no presente estudo nas análises de cromatografia gasosa executadas na silagem ácida de camarão e nas rações com elas formuladas.

Espécies de água doce possuem enzimas capazes de alongar e dessaturar ácidos graxos precursores de ácidos graxos essenciais, além de terem habilidade para utilizar lipídios com altos níveis de ácidos graxos livres (BAHURMIZ et al., 2007; MARTINO \& TAKAHASHI, 2001). Os resultados encontrados no tecido muscular das tilápias analisadas comprovaram a incorporação de 10\% (EPA + DHA) em relação ao tratamento controle, quando a adição de silagem na ração foi maior (T5=16\%), aumentando em 
Tabela 2 - Lipídios totais, ácido eicosapentaenóico (20:5n-3) e ácido docosahexanóico (22:6n-3) das dietas com diferentes níveis de adição de silagem ácida de cabeça do resíduo do camarão, encontrados nos filés de tilápias submetidas às dietas e peso final (g) das tilápias aos 60 dias de cultivo.

\begin{tabular}{|c|c|c|c|c|c|}
\hline & 0 & 4 & 8 & 12 & 16 \\
\hline Dietas $_{\mathrm{LT}^{2}}$ & $9,95 \pm 0,96$ & $8,75 \pm 0,41$ & $10,30 \pm 0,68$ & $6,70 \pm 0,06$ & $7,03 \pm 0,02$ \\
\hline $\mathrm{EPA}^{4}$ & ND & 38,43 & 50,62 & 69,84 & 68,81 \\
\hline $\mathrm{DHA}^{4}$ & ND & 31,72 & 43,69 & 53,78 & 67,72 \\
\hline $\mathrm{PF}^{3}$ & $76,07 \pm 18,2$ & $77,06 \pm 28,9$ & $79,10 \pm 7,78$ & $71,96 \pm 12,1$ & $61,44 \pm 25,7$ \\
\hline $\mathrm{LT}^{2}$ & $1,37 \pm 0,19$ & $1,24 \pm 0,25$ & $0,99 \pm 0,42$ & $0,79 \pm 0,21$ & $0,79 \pm 0,14$ \\
\hline $\mathrm{EPA}^{4}$ & ND & ND & ND & 0,95 & 1,34 \\
\hline $\mathrm{DHA}^{4}$ & 11,14 & 19,45 & 16,23 & 25,92 & 40,50 \\
\hline
\end{tabular}

${ }^{1}$ Os valores são médias de duplicatas \pm desvio padrão.

${ }^{2} \mathrm{LT}=$ Lipídios Totais (\% da amostra úmida).

${ }^{3} \mathrm{PF}=$ Peso final das tilápias $(\mathrm{g})$

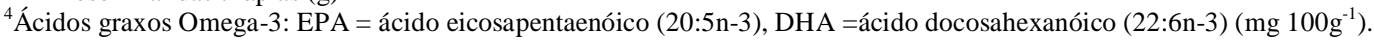

$\mathrm{ND}=\mathrm{Não}$ Detectado.

LT dietas: $Y=4,16-0,095375 x\left(R^{2}=99,05 \%\right)$; EPA dietas: $Y=0,09576 x+0,64\left(R^{2}=99,70 \%\right)$; DHA dietas: $Y=0,242625 x-0,452\left(R^{2}=91,97 \%\right)$. $(\mathrm{P}<0,05)$.

LT tilápias: $\mathrm{Y}=1,356-0,040250 \mathrm{x}\left(\mathrm{R}^{2}=93,96 \%\right)$; EPA tilápias: $\mathrm{Y}=0,0041 \mathrm{x}^{2}-0,0288 \mathrm{x}+0,012\left(\mathrm{R}^{2}=98,65 \%\right)$; DHA tilápias: $\mathrm{Y}=-0,001 \mathrm{x}^{2}+0,4152 \mathrm{x}+2,4806\left(\mathrm{R}^{2}=89,09 \%\right) ; \mathrm{PF}: \mathrm{Y}=-2,30 \mathrm{x}^{2}+10,365 \mathrm{x}+67,333\left(\mathrm{R}^{2}=97,16 \%\right)$.

mais de três vezes os teores obtidos com dietas suplementadas com óleos vegetais (MARTINO \& TAKAHASHI, 2001). Entretanto, as tilápias não apresentaram crescimento linear significativo com a inclusão dos níveis de silagem nas dietas, corroborando os resultados encontrados por COSTA et al. (2009).

Embora peixes de água doce sejam caracterizados por conter em sua composição natural alta concentração de ácidos graxos ômega-6, principalmente ácidos araquidônico e linoleico, o fornecimento de dietas contendo óleo de peixe a carpas e salmonídeos aumenta o teor de ômega-3 na composição do filé desses peixes (STEFFENS, 1997). Esses dados confirmam que a formulação da dieta é fator fundamental na composição do tecido do peixe e que peixes de água doce possuem naturalmente baixos teores de ácidos graxos ômega-3, diferentemente das espécies marinhas (HOAR et al., 1979).

A modernização na confecção industrial dos alimentos e a melhoria da composição das dietas de animais são procedimentos muito eficazes na prevenção da deficiência de alguns nutrientes essenciais e na incorporação de compostos ativos não presentes nas fontes de alimentos, como o ômega-3 em tilápias. Segundo OLSEN \& SECHER (2002), peixes marinhos são reconhecidos como um dos alimentos mais importantes para dieta e nutrição humana, devido à presença de EPA e DHA em sua constituição. Com a possibilidade da incorporação desses ácidos graxos essenciais no filé de tilápias, o presente trabalho pode se tornar uma estratégia alimentar importante para os órgãos ligados à saúde humana.

Com o intuito de reduzir o risco de doenças cardiovasculares, as organizações de saúde de vários países recomendam uma ingestão diária em torno de 1,2-2,0 $\mathrm{g} \mathrm{dia}^{-1}$ de ácidos graxos poliinsaturados (ômega-3), independente do sexo, idade e hábito alimentar das pessoas (IZQUIERDO et al., 2005). Nesse sentido, estudos têm relacionado a redução da mortalidade por doenças cardiovasculares com o consumo frequente de óleo de peixe marinho na dieta (STEFFENS, 1997).

Com a depleção dos estoques pesqueiros e consequente limitado fornecimento de óleos e farinha de carne de peixes marinhos destinados a rações, surgiu a necessidade de se encontrar substitutos para a manutenção e produção de rações para aquicultura. Nesse sentido, a silagem de cabeça de camarão aponta ser uma alternativa na dieta de organismos aquáticos, como fonte rica em ácidos graxos poliinsaturados ômega-3, especialmente de DHA, corroborando BRUM et al. (2002); MARTINO \& PORTZ (2006). Com isso, a utilização de silagem de camarão na alimentação de tilápias torna-se viável para a incorporação de ômega3 nos filés, além de fornecer uma alternativa para o destino do resíduo de indústrias beneficiadoras de camarão, minimizando assim também problemas ambientais. 


\section{CONCLUSÃO}

A inclusão de $16 \%$ de silagem ácida de cabeça de camarão como fonte alternativa de ácidos graxos essenciais em dietas para tilápia aumenta os níveis de EPA e DHA no seu filé.

\section{AGRADECIMENTOS}

À Coordenação de Aperfeiçoamento de Pessoal de Nível Superior (CAPES), pela concessão da bolsa de estudos, à FAPESB, pelo recurso financeiro, à Bahia Pesca.

\section{REFERÊNCIAS}

AIURA, F.S.; CARVALHO, M.R.B. Composição em ácidos graxos e rendimento de filé de tilápia do Nilo (Oreochromis niloticus) alimentada com dietas contendo tanino. Revista Portuguesa de Ciências Veterinárias, v.550, p.93-98, 2004. Disponível em: <http://www.fmv.utl.pt/spcv/PDF/pdf6_2004/ 550_93_98.pdf>. Acesso em: 29 jun. 2007.

BAHURMIZ, O.M.; NG, W.K. Effects of dietary palm oil source on growth, tissue fatty acid composition and nutrient digestibility of red hybrid tilapia, Oreochromis sp., raised from stocking to marketable size. Aquaculture, v.262, p.382-392, 2007. Disponível em: <http://www.sciencedirect.com/science/ article/pii/S0044848606008921>. Acesso em: 01 jul. 2007. doi: $10.1016 /$ S0044848606008921.

BLIGH, E.G.; DYER, W.J. A rapid method of total lipid extraction and purification. Canadian Journal Biochemistry and Physiology, v.37, n.8, p.911-917, 1959.

BORGHESI, R. Avaliação físico-química, nutricional e biológica das silagens ácida, biológica e enzimática elaboradas com descarte e resíduo do beneficiamento da tilápia do Nilo (Oreochromis niloticus). 2004. 108f. Dissertação (Mestrado em Ciência e Tecnologia de Alimentos) - Escola Superior de Agricultura "Luiz de Queiroz", Universidade de São Paulo, SP.

BRUM, A.A.S. et al. Óleo de peixe como suplemento dietético. Revista de Ciência e Tecnologia, v.10, n.19, p.71-78, 2002.

CAVALHEIRO, J.M.O. et al. Utilization of shrimp industry waste in the formulation of tilápia (Oreochromis niloticus Linnaeus) feed. Bioresource Technology, v.98, p.602-606, 2007. Disponível em: <http://www.sciencedirect.com/science/ article/pii/S096085240600068X>. Acesso em: 26 abr. 2006. doi:10.1016/096085240600068X.

COSTA, C.N. Avaliação da silagem ácida do resíduo do camarão branco (Litopenaeus vannamei) como fonte protéica na alimentação da tilápia do nilo (Oreochromis niloticus). 2007. 45f. Dissertação (Mestrado em Ciências Agrárias) Centro de Ciências Agrárias, Ambientais e Biológicas, Universidade Federal do Recôncavo da Bahia, BA.

COSTA, C.N. et al. Silagem ácida do resíduo do camarão Litopenaeus vannamei em rações para tilápia do Nilo. ActaScientiarum. Animal Sciences, v.31, p.161-167, 2009. Disponível em: <http://periodicos.uem.br/ojs/index.php/ ActaSciAnimSci/article/view/5097/5097>. Acesso em: 25 jul. 2008. doi: 10.4025/actascianimsci.v31i2.5097.
DAVID, R. Increasing omega-3 fatty acid consumption through food fortification. British Food Journal, v.95, n.5, p.30-32, 1993.

FAGBENRO, O.A.; BELLO-OLUSOJI, O.A. Preparation, nutrient composition and digestibility of fermented shrimp head silage. Food Chemistry, v.60, n.4, p.489-493, 1997. Disponível em: <http://www.sciencedirect.com/science/article/ pii/S0308814696003147>. Acesso em: 03 fev. 2006. doi: $10.1016 /$ S0308814696003147.

FERREIRA, D.F. SISVAR: sistema de análise de variância para dados balanceados, versão 4.0. Lavras: DEX/UFLA, 1999. (Software estatística).

FURUYA W.M. et al. Composição centesimal e perfil de ácidos graxos do camarão-d’água-doce. Revista Brasileira de Zootecnia, v.35, n.4, p.1577-1580, 2006. Disponível em: <http://www.scielo.br/pdf/rbz/v35n4s0/a01v354s.pdf〉. Acesso em: 18 jan. 2007.

HOAR, W.S. et al. Fish physiology. New York: Academic, 1979. V.8, 786p.

HUANG, C.H. et al. Characteristics of lipid peroxidation in sarcoplasmic reticulum of tilapia. Food Science, v.25, p.104$108,1998$.

IZQUIERDO, M.S. et al. Alterations in fillet fatty acid profile and flesh quality in gilthead seabream (Sparus aurata) fed vegetable oils for a long term period. Recovery of fatty acid profiles by fish oil feeding. Aquaculture, v.250, p.431-444, 2005. Disponível em: <http://www.sciencedirect.com/science/ article/pii/S0044848604007021>. Acesso em: 01 set. 2009. doi:10.1016/ S0044848604007021.

JOSEPH, J.D.; ACKMAN, R.G. Capillary column gas chromatography method for analysis of encapsulated fish oil and fish oil ethyl esters: collaborative study. Journal AOAC International, v.75, p.488-506, 1992.

KRZYNOWEK, J.; PANUNZIO, L.J. Cholesterol and fatty acids in several species of shrimp. Journal of Food Science, v.54, n.2, p.237-239, 1989.

MARTINO, R.C.; PORTZ, L. Estratégias para desenvolvimento de rações para peixes carnívoros de água doce: fontes de proteína e lipídios. Tópicos Especiais em Biologia Aquática e Aqüicultura, Aquaciência 2004, p.125-138, 2006.

MARTINO, R.; TAKAHASHI, N.S. A importância da adição de lipídios em rações para a aqüicultura. Óleos e Grãos, v.58, p.32-37, 2001.

MPA- Ministério da Pesca e Aquicultura. Produção Pesqueira e Aquícola-Estatística 2008 e 2009. Brasília, 2010. 30f.

OLSEN, S.F., SECHER, N.J. Low consumption of seafood in early pregnancy as a risk factor for preterm delivery: prospective cohort study. British Medical Journal, v.324, p.1-5, 2002.

OM, A.D. et al. The effects of dietary EPA and DHA fortification on lipolysis activity and physiological function in juvenile black sea bream Acanthopagrus schlegeli (Bleeker). Aquaculture Research, v.32, Suppl.1, p.255-262, 2001. 
PEZZATO, L.E. et al. Avaliação de dois métodos de determinação do coeficiente de digestibilidade aparente com a tilápia do Nilo (Oreochromis niloticus L.). Acta Scientiarum, v.24, n.4, p.965-971, 2002. Disponível em: <http:// periodicos.uem.br/ojs/index.php/ActaSciAnimSci/article/view/ 2452/1732>. Acesso em: 29 jan. 2007.

ROSENLUND, G. et al. Effect of alternative lipid sources on long-term growth performance and quality of Atlantic salmon (Salmo salar L.). Aquaculture Research, v.32 (Suppl.1), p.323-328, 2001.

SRIKET, P, et al. Comparative studies on chemical composition and thermal properties of black tiger shrimp (Penaeus monodon) and white shrimp (Penaeus vannamei) meats. Food Chemistry, v.103, p.1199-1207, 2007. Disponível em: <http:/ / w w w s s iencedirect.com/science/article/pi i/ S0308814606008703>. Acesso em: 23 nov. 2009. doi:10.1016/ S0308814606008703.
STEFFENS, W. Effects of variation in essential fatty acids in fish feeds on nutritive value of fresh water fish for humans. Aquaculture, v.151, p.97-119, 1997. Disponível em: <http:/ / w ww.sciencedirect.com/science/article/pi i / S0044848696014937>. Acesso em: 18 nov. 2009. doi: $10.1016 /$ S0044848696014937.

VILA NOVA, C.M.V.M. et al. Composição química, teor de colesterol e caracterização dos lipídios totais de tilápia (Oreochromis niloticus) e pargo (Lutjanus purpureus). Ciência e Tecnologia de Alimentos, v.25, n.3, p.430-436, 2005. Disponível em: <http://dx.doi.org/10.1590/S010120612005000300006>. Acesso em: 12 jan. 2007. doi: 10.1590/ S0101-20612005000300006.

VISENTAINER, J.V. et al. Relação entre teores de colesterol em filés de tilápias e níveis de óleo de linhaça na ração. Ciência e Tecnologia de Alimentos, v.25, n.2, p.310-314, 2005. Disponível em: <http://www.scielo.br/pdf/cta/v25n2/25030.pdf>. Acesso em: 01 dez. 2006. 